\title{
Composición del Fitoplancton en el embalse Los Laureles, Francisco Morazán, Honduras
}

Jorge Luis Carranza Sánchez ${ }^{1}$

Alba Isbela Hernández Oviedo ${ }^{2}$

\section{RESUMEN}

El Embalse Los Laureles es un ecosistema artificial creado para abastecimiento de agua potable. Se estudiaron los parámetros físicos (temperatura, conductividad, turbidez y disco Secchi), químicos (pH, oxígeno disuelto,) y biológicos (clorofila a), con el objetivo de determinar la composición (abundancia y diversidad) del fitoplancton durante marzo-agosto 2014. Se obtuvieron muestras biológicas en tres puntos del embalse que comprenden cortina, centro y cola con $100 \mathrm{~mL}$, utilizando red de arrastre a nivel sub superficial $20-30 \mathrm{~cm}$ de profundidad durante dos minutos para análisis cualitativo y con botella de Van Dorn a profundidad Secchi para análisis cuantitativo. Los perfiles clinógrados de oxígeno, turbidez elevada en el hipolimnion, baja transparencia de Secchi persistentes en todos los muestreos, 21 taxa identificadas, de las cuales 7 pertenecen a las Cianofíceas, 5 a las Bacillariophyceas y 9 a las Clorofíceas. Durante el tiempo de muestreo, las Cianofíceas se encontraron en un $67 \%$, las Bacillariophyceas en un $26 \%$ y Clorofíceas en un $7 \%$, estos factores indican características de cuerpos de agua eutróficos. Sin embargo, para obtener resultados con mayor grado de confiabilidad, se recomienda realizar los muestreos físicos, químicos, biológicos y de productividad por lo menos cada quince días durante la época seca y lluviosa, aumentando los muestreos cuando se sospecha de floraciones algales, ya que estas relaciones son más precisas cuando los estudios se realizan por ciclos anuales, para identificar patrones de variación y tendencias que ayuden a prevenir y corregir este problema.

Palabras clave: fitoplancton, composición, diversidad, cianofíceas

\footnotetext{
${ }^{1}$ Estudiante de Biología. Laboratorio de Hidrobiología. Facultad de Ciencia, UNAH. jorge_ 900707@hotmail.com

${ }^{2}$ Asesora. Profesora de la Escuela de Biología, Facultad de Ciencias UNAH. Correo albaoviedo@yahoo.com
} 


\section{ABSTRACT}

The Laureles reservoir is an artificial ecosystem created for drinking water. The composition (abundance and diversity) of phytoplankton along with the physical parameters (temperature, conductivity, turbidity and Secchi disk), chemical ( $\mathrm{pH}$, dissolved oxygen) and biological (chlorophyll a) all during March-August 2014 was studied. Samples in three sampling points comprising curtain, middle and tail with $100 \mathrm{~mL}$ were obtained using trawl subsurface level 20 to $30 \mathrm{~cm}$ deep for two minutes to qualitative analysis and Van Dorn bottle for Secchi depth quantitative analysis.

Of the 21 taxa identified 7 belong to the cyanobacteria, diatoms and 5 to 9 at Chlorophycea. During the sampling time, the cyanobacteria were found in $67 \%$, diatoms by $26 \%$ and $7 \%$ Chlorophyceae, it clinógrados profiles with oxygen and $\mathrm{pH}$, high turbidity in the hypolimnion, persistent low Secchi transparency in all samples, and trophic state index data of a TSI 2011 for Secchi disk 58, 80 to 68 for total phosphorus and chlorophyll a, TSI values $>54$ are to eutrophic lakes. All these factors indicate characteristics of eutrophic water bodies. However, for results with a higher degree of reliability, it is recommended that the physical, chemical, biological and productivity samplings at least every two weeks during the dry and rainy seasons, increasing the sampling when suspected algal blooms, as these relationships are more accurate when studies are performed yearly cycles, to identify patterns of variation and trends to help prevent and correct this problem.

Keywords: phytoplankton, composition, diversity, Cyanophyceae. 


\section{INTRODUCCIÓN}

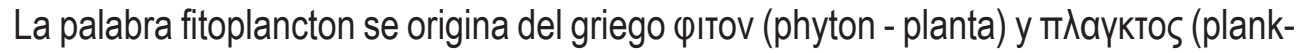
tos - errante). Es una comunidad de organismos microscópicos fotosintetizadores que se encuentran suspendidos en la zona fótica de la columna de agua (Reynolds, 1984; Kilham y Hecky, 1988, en Martínez, Ortega y Ramos, 2014). El fitoplancton desempeña un rol muy importante como base de la cadena alimenticia y como indicadores de la calidad del agua (Martínez, et al., 2014).

El fitoplancton de aguas continentales se desarrolla en ambientes lénticos que comprenden aguas estancadas como lagos, lagunas y embalses; en ambientes lóticos de agua corriente, como los manantiales, ríos, arroyos, cascadas y canales. El fitoplancton por lo general presenta un ciclo anual previsible, sin embargo algunas especies, como las cianobacterias, pueden proliferar excesivamente y crear "florecimientos", los cuales llegan a ser potencialmente tóxicos provocando en el agua olor y sabor desagradable (Vaulot, 2006, en Martínez et al., 2014).

De acuerdo con Smith y Smith (2001), composición de especies es el término que se emplea para incluir tanto el número de especies, como su abundancia relativa que se puede obtener contando todos los individuos de cada especie en una serie de puntos de muestreo, definiendo así la estructura biológica de una comunidad.

En las últimas décadas el interés por el estudio del fitoplancton presente en lagos y embalses se ha incrementado significativamente. Debido a la problemática de eutrofización definida por la excesiva cantidad de nutrientes presente en el sistema en particular de nitrógeno y fósforo, el desarrollo de floraciones algales particularmente de cianobacterias y la escasa transparencia del cuerpo de agua, son algunos de los principales efectos de esta problemática (Rodríguez et al. 2008).

Según Quirós (2004), en países como Argentina y Uruguay las cianobacterias comenzaron a verse como un problema de grave perjuicio para la calidad del agua y la salud humana, señalan que a mayores concentraciones de nutrientes y menores profundidades los lagos y los embalses presentan mayor biomasa algal.

Debido a lo mencionado anteriormente, autores como Vila, Ilse y Montecino (1987), aluden que el conocimiento taxonómico y ecológico del fitoplancton en cuerpos artificiales de agua, tales como los embalses se necesita para comprender el comportamiento y el impacto de estos organismos a través del tiempo. 
Por su parte el embalse Los Laureles constituye una obra de represamiento en la Subcuenca del Río Guacerique, según Tavera (2013), este embalse es el más vulnerable hoy en día, ya que está sujeto a presiones de urbanización incontrolada en sus alrededores, de contaminación y sedimentación en su fondo, limitando con esto la capacidad de almacenamiento del recurso hídrico.

El presente estudio se realizó con los siguientes objetivos: Medir los parámetros físicos (temperatura, conductividad, turbidez y disco Secchi), químicos ( $\mathrm{pH}$, oxígeno disuelto,) y biológico (clorofila a).

Identificar y cuantificar los organismos fitoplanctónicos que influyen en la calidad del agua del Embalse Los Laureles.

Además, con los resultados de este trabajo se pretende que las autoridades gubernamentales presten atención al problema de las algas tóxicas que pueden ocasionar problemas en la salud, en cuerpos de agua tan fundamentales como son los embalses, por ser reservorios de agua que se destina a la potabilización. Según Luna, Díaz y Rodriguez (2010), las cianobacterias que emiten toxinas pueden ocasionar brotes agudos de gastroenteritis, congestión pulmonar, irritaciones cutáneas, alergias, lesiones hepáticas, e incluso, elevación en la incidencia de tumores.

\section{Área de estudio}

El Embalse Los Laureles se encuentra en la Subcuenca del Río Guacerique, al suroeste de Tegucigalpa en las coordenadas $14^{\circ} 03^{\prime} 40^{\prime \prime}$ y $14^{\circ} 06^{\prime} 40^{\prime \prime}$ latitud norte, y $87^{\circ} 14^{\prime} 51^{\prime \prime}$ y $87^{\circ} 14^{\prime} 23^{\prime \prime}$ longitud oeste. La Subcuenca tiene una extensión aproximada de $210 \mathrm{~km}^{2}$, mientras que el embalse tiene un área de aproximadamente $1 \mathrm{~km}^{2}$. El embalse fue construido en los años 70 (1976-1978), por parte del Banco Interamericano de Desarrollo (BID) (Tavera, 2013).

\section{Material y métodos}

Se seleccionaron tres estaciones de muestreo denominadas cortina, centro y cola (Figura 1). Las muestras se tomaron con una frecuencia quincenal entre marzo y agosto del 2014, y semanal con la presencia de afloramientos, ya que antes de las primeras lluvias de invierno se presentó este evento.

En cada estación se midió la temperatura, conductividad, turbídez, y la transparencia del disco Secchi, pH y oxígeno disuelto por cada metro de profundidad. También se 
tomaron muestras para análisis de clorofila a (1000 mL), el cual se realizó con un volumen de filtrado de 50 a $200 \mathrm{~mL}$ dependiendo de la concentración de algas en muestra, el protocolo utilizado fue el siguiente:

1.Filtración en bomba de vacío eléctrica (filtro de $47 \mathrm{~mm}$ y $0.45 \mu \mathrm{m}$ ).

2.Macerado.

3. Preservación de la muestra por 24 horas con $10 \mathrm{~mL}$ de acetona al $90 \%$, en tubos de ensayo cubiertos con papel aluminio.

4.Centrifugación por 20 minutos.

5.Lectura en espectrofotómetro.

Figura 1. Embalse Los Laureles puntos de muestreo.

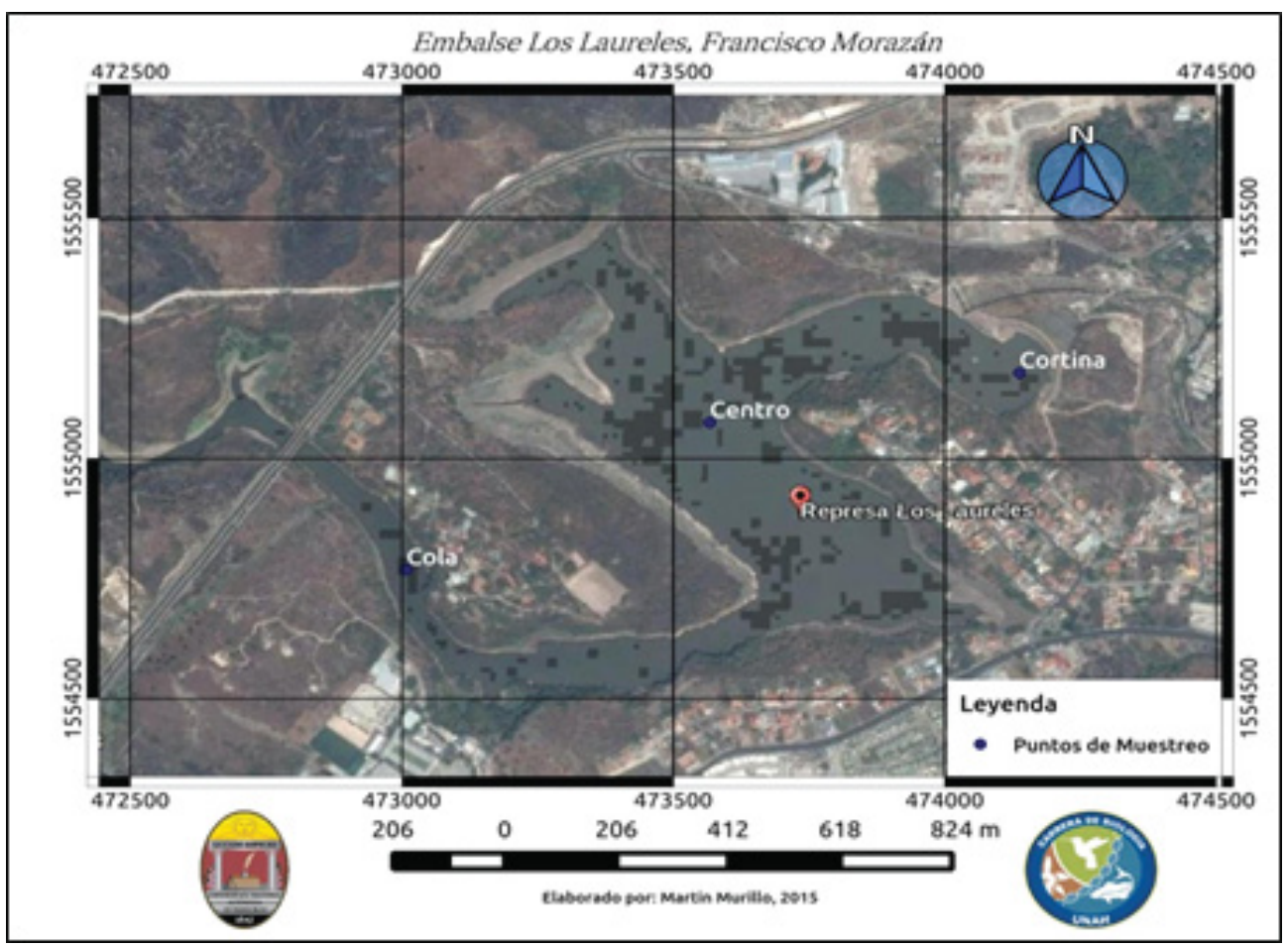

Fuente: Murillo,2015

Muestras de $100 \mathrm{~mL}$ para análisis cualitativo de fitoplancton mediante arrastre con una red de plancton durante dos minutos a nivel sub superficial $20-30 \mathrm{~cm}$ y para análisis cuantitativo $(100 \mathrm{~mL})$ con botella de Van Dorn a profundidad Secchi. Las muestras cualitativas y cuantitativas se fijaron in situ con lugol al $10 \%$ del volumen de la muestra. 
Para el análisis de diversidad se realizó la identificación con microscopio óptico a 10x, 40x y 100x, se trabajó con un volumen de $0.14 \mathrm{~mL}$ (3 gotas) por muestra y, para la abundancia el recuento se hizo a 10x y 40x usando un contador de células donde se contabilizó un volumen de $0.19 \mathrm{~mL}$ (4 gotas) por muestra sin el uso de cámaras de conteo, dando un aproximado en células/mL, (Moreno, Medina y Albarracín, 2012).

También se utilizaron las claves taxonómicas de Novelo (2011); Novelo (2012a), Novelo (2012b), Parra, et al., (1983a,) Parra, et. al., (1983b), UNESCO (2009), Whitford y Schumacher (1984). Los organismos se identificaron a nivel de genero considerándose como tal una colonia, un filamento o una célula siguiendo el trabajo de De León y Chalar, (2003).

Se empleó el Índice de diversidad de Shannon (en Smith y Smith, 2001). Este índice se expresa con un número positivo, que en la mayoría de los ecosistemas naturales varía entre 1 y 5 (Emanuelli, 2010).

El porcentaje de similitud (PS) de Sorensen en el que se tabula la abundancia de especies en cada punto de muestreo como un porcentaje (en Smith y Smith, 2001).

\section{Resultados}

Parámetros físicos y químicos

La temperatura mínima del agua a profundidad Secchi $0.50 \mathrm{~m}$ se registró en marzo con $24^{\circ} \mathrm{C}$ aumentando hasta alcanzar el máximo en el mes de abril con $26^{\circ} \mathrm{C}$ a $0.47 \mathrm{~m}$ Secchi, llegando en julio a $25^{\circ} \mathrm{C}$ a $0.43 \mathrm{~m}$ Secchi.

Para la conductividad en $\mu \mathrm{S} / \mathrm{cm}$ véase la Figura 2. La turbidez se puede apreciar en la Figura 3.

El oxígeno disuelto uno de los parámetros Químicos más importantes para la calidad del agua, en las tres estaciones de muestreo presentó un perfil clinógrado durante los cinco meses del estudio (Figura 4).

En cuanto al pH a profundidad de disco Secchi el valor más bajo se presentó en el mes de abril con 6.4 y el más alto se registró en julio con 8.7 . 
Figura 2. Perfil de conductividad. Los valores más altos se observan en la cortina debido al mayor contenido en materiales minerales que ingresan por este sitio

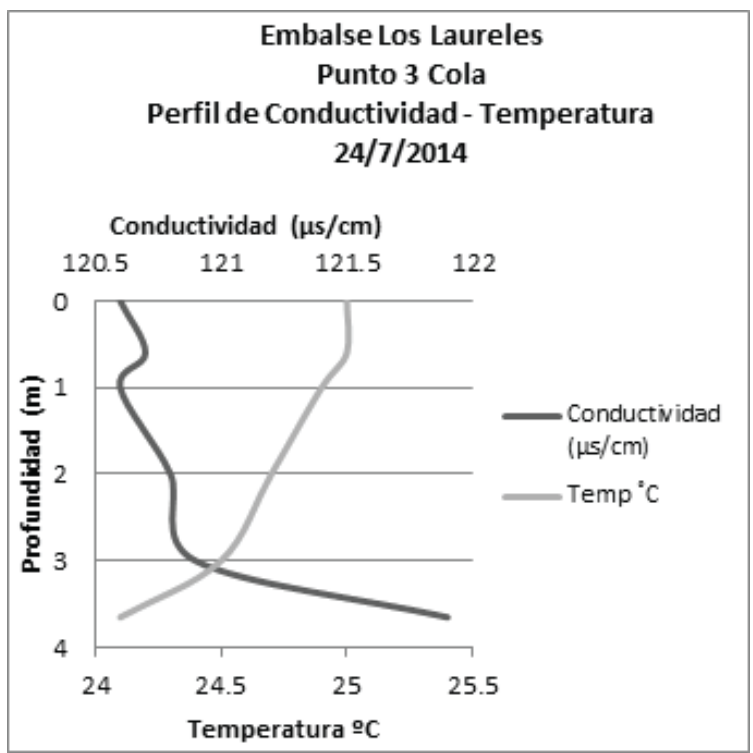

Fuente: elaboración propia.

Figura 3. Perfil de turbidez. Se pueden apreciar que la turbidez aumenta conforme se acerca al hipolimnion

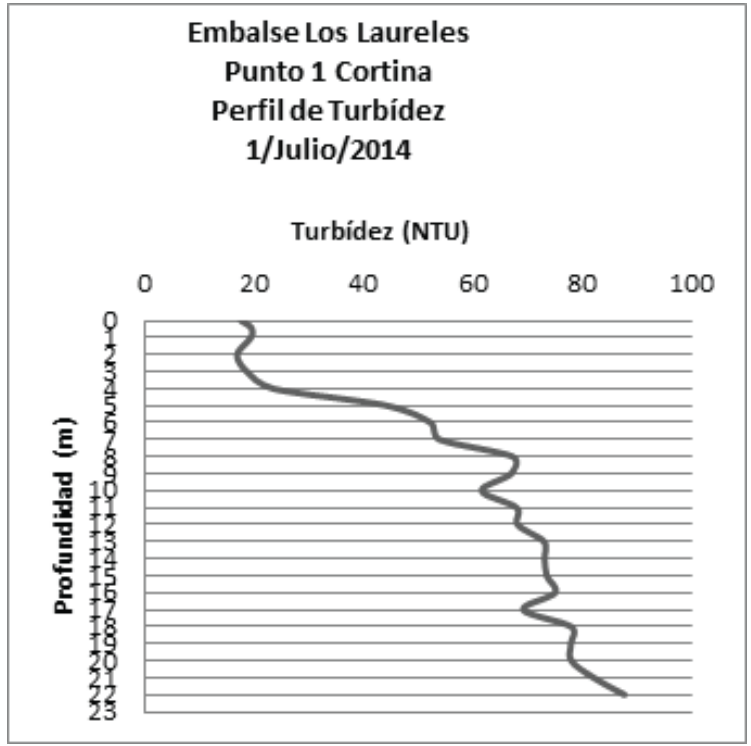

Fuente: elaboración propia. 
Figura 4. Perfil de oxígeno disuelto - temperatura. Se presentan perfiles clinógrados

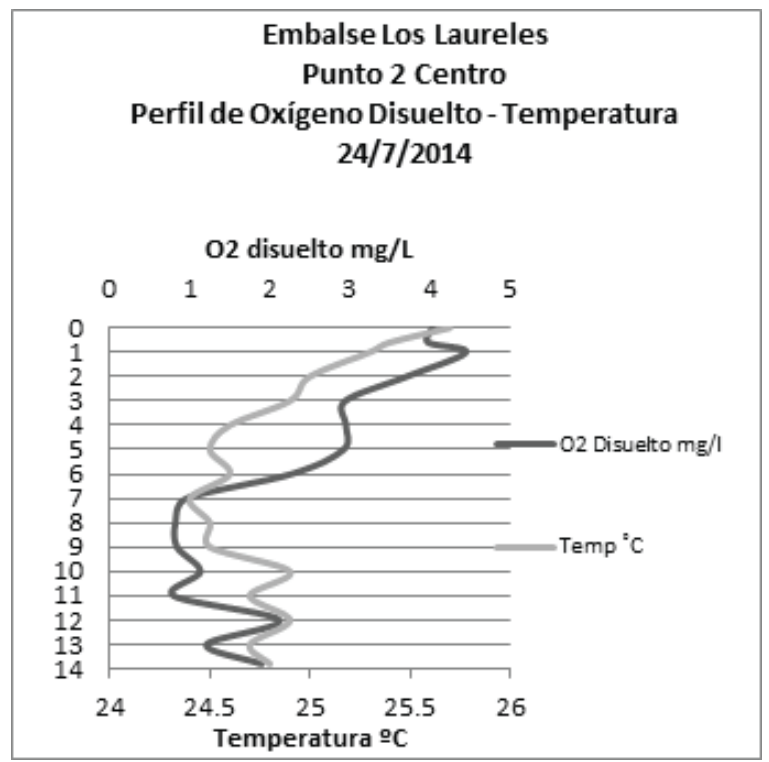

Fuente: elaboración propia.

\section{Biomasa del fitoplancton}

La biomasa del fitoplancton, medida como la concentración de clorofila a, registró un valor promedio de $27.72 \mathrm{mg} / \mathrm{m}^{3}$ para las 3 estaciones en los cinco meses analizados. Los menores valores se presentaron en marzo con $3.02 \mathrm{mg} / \mathrm{m}^{3}$ para la cortina, y la mayor concentración se registró en abril con $57.62 \mathrm{mg} / \mathrm{m}^{3}$ en la cortina, dicho aumento coincidió con el afloramiento de Cianofíceas (Figura 5).

Composición taxonómica del fitoplancton

De los 21 taxa identificados 7 pertenecen a las Cianofíceas (67\%), 5 a las Bacillariophyceas $(26 \%)$ y 9 a las Clorofíceas $(7 \%)$.

Variación espacial y temporal del fitoplancton

Durante los cinco meses se realizó un total de once muestreos y espacialmente la mayor abundancia se presentó en la estación cortina, durante el verano (Tabla 1). Mientras que, temporalmente la mayor diversidad se registró en julio (Tabla 2). Por su parte el género que se encontró durante todos los muestreos fue Anabaena sp del grupo de las Cianofíceas (Figura 5). 
Tabla 1. Fitoplancton identificado y cuantificado en un afloramiento en la cortina el 29 abril de 2014

\begin{tabular}{|l|c|c|c|c|c|}
\hline \multicolumn{2}{|c}{ Clase } & Familia & Género & Especie & Cortina Cél/ml. \\
\hline Cyanoprokaryota & Cyanophyceae & Nostocaceae & $\begin{array}{c}\text { Anabaena, } \\
\text { Bory 1822 }\end{array}$ & Anabaena sp. & 19,407 \\
Bacillariophyta & Coscinodiscophyceae & $\begin{array}{c}\text { Aulacoseiraceae } \\
\text { Crawford } \\
\text { Aulacoseira } \\
\text { Thwai-tes } \\
\text { Melosiraceae, } \\
\text { Kützing 1844 }\end{array}$ & $\begin{array}{c}\text { Melosira, } \\
\text { Agardh 1824 }\end{array}$ & Aulacoseira sp & 200 \\
\hline
\end{tabular}

Fuente: elaboración propia

Figura 5. Variación de Anabaena sp. de marzo-agosto 2014. Nótese que para el 24 de abril y el 12 de agosto las cantidades empiezan a incrementar, esto coincide con los periodos previos a las primeras lluvias de invierno

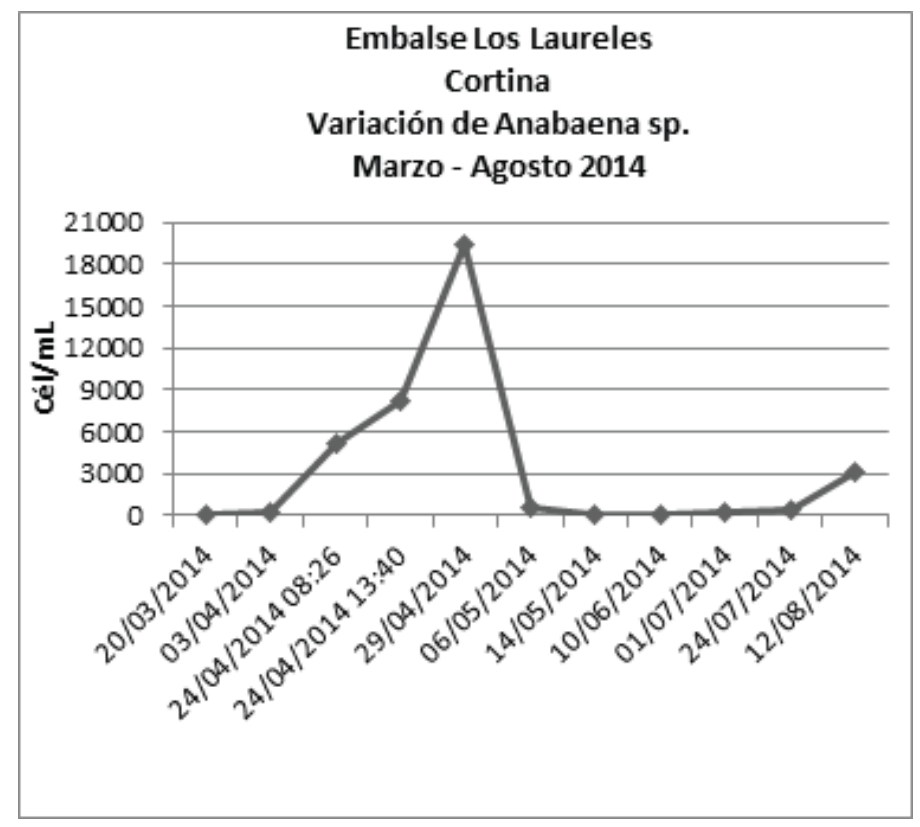

Fuente: elaboración propia 
Tabla 2. Fitoplancton identificado y cuantificado para el 1 de julio de 2014

\begin{tabular}{|c|c|c|c|c|c|c|c|}
\hline Filo & Clase & Familia & Cénero & Especie & $\begin{array}{l}\text { Cortina } \\
\text { Cél/mL }\end{array}$ & $\begin{array}{l}\text { Centro } \\
\text { Cél/mL }\end{array}$ & $\begin{array}{c}\text { Cola } \\
\text { Cél/mL }\end{array}$ \\
\hline \multirow[t]{7}{*}{ Cyanoprokaryota } & $\begin{array}{l}\text { Cyanophy- } \\
\text { ceae }\end{array}$ & Nostoca-ceae & $\begin{array}{l}\text { Anabaena, Bory } \\
1822\end{array}$ & Anabaena sp. & 157 & 200 & 1105 \\
\hline & & & $\begin{array}{l}\text { Nostoc Vaucher } \\
\text { ex Bornet \& } \\
\text { Flahault } 1886\end{array}$ & Nostoc sp. & 26 & 15 & 73 \\
\hline & & & Cuspidothrix & Cuspidothrix sp. & 100 & 15 & 73 \\
\hline & & & $\begin{array}{l}\text { Cylindros- } \\
\text { permopsis }\end{array}$ & $\begin{array}{l}\text { Cylindrospermop- } \\
\text { sis sp. }\end{array}$ & 26 & 31 & 57 \\
\hline & & $\begin{array}{l}\text { Pseuda- } \\
\text { nabaena-ceae }\end{array}$ & Pseudana-baena & $\begin{array}{l}\text { Pseudanabaena } \\
\text { sp. }\end{array}$ & 126 & 157 & 226 \\
\hline & & $\begin{array}{l}\text { Oscillato- } \\
\text { riaceae }\end{array}$ & $\begin{array}{l}\text { Lyngbya Agardh } \\
\text { ex Gomont }\end{array}$ & Lyngbya sp. & 15 & 5 & 126 \\
\hline & & $\begin{array}{l}\text { Mycrocys- } \\
\text { taceae }\end{array}$ & $\begin{array}{l}\text { Microcystis } \\
\text { Kützing }\end{array}$ & Microcystis sp. & 36 & 10 & 63 \\
\hline \multirow[t]{5}{*}{ Chlorophyta } & $\begin{array}{l}\text { Zygnemato- } \\
\text { phyceae }\end{array}$ & Closte-riaceae & $\begin{array}{l}\text { Closterium } \\
\text { Nitzsch ex Ralfs } \\
1848\end{array}$ & Closterium sp.2 & 52 & 42 & 78 \\
\hline & & Desmi-diaceae & $\begin{array}{l}\text { Staurastrum } \\
\text { Meyen ex Ralfs } \\
1848\end{array}$ & Staurastrum sp. & 26 & 10 & 21 \\
\hline & $\begin{array}{l}\text { Chlorophy- } \\
\text { ceae }\end{array}$ & Palmella-ceae & $\begin{array}{l}\text { Sphaerocystis } \\
\text { Chodat } 1897\end{array}$ & Sphaerocystis sp. & 15 & 26 & 10 \\
\hline & & Oocysta-ceae & $\begin{array}{l}\text { Ankistrodes-mus } \\
\text { Corda } 1838\end{array}$ & $\begin{array}{l}\text { Ankistrodesmus } \\
\text { sp. }\end{array}$ & 21 & 10 & 31 \\
\hline & & Chlorella-ceae & $\begin{array}{l}\text { Chlorella } \\
\text { Beijerinck } 1890\end{array}$ & Chlorella sp. & 0 & 89 & 1421 \\
\hline \multirow[t]{3}{*}{ Bacillariophyta } & $\begin{array}{l}\text { Coscinodis- } \\
\text { cophyceae }\end{array}$ & $\begin{array}{l}\text { Aulaco- } \\
\text { seiraceae } \\
\text { Crawford }\end{array}$ & $\begin{array}{l}\text { Aulacoseira } \\
\text { Thwaites }\end{array}$ & Aulacoseira sp. & 163 & 115 & 436 \\
\hline & & Melosira-ceae & $\begin{array}{l}\text { Melosira Agardh } \\
1824\end{array}$ & Melosira sp. & 57 & 26 & 100 \\
\hline & & & & Nitzschia sp. & 0 & 0 & 10 \\
\hline
\end{tabular}

Fuente: elaboración propia 
En la Tabla 3 y 4 se pueden observar los valores para el índice de diversidad de Shannon y porcentaje de similitud de Sorensen respectivamente.

Tabla 3. Valores del índice de Shannon marzo - julio 2014

\begin{tabular}{|l|l|l|l|}
\hline Fecha & Cortina & Centro & Cola \\
\hline $20 / 3 / 2014$ & 1.45 & 1.47 & 0.91 \\
\hline $3 / 4 / 2014$ & 1.44 & 1.57 & 1.18 \\
\hline $24 / 4 / 20148: 26 \mathrm{am}$ & 1.58 & -- & 0.89 \\
\hline $24 / 4 / 20141: 40 \mathrm{pm}$ & 0.11 & -- & -- \\
\hline $29 / 4 / 2014$ & 0.09 & -- & -- \\
\hline $6 / 5 / 2014$ & 0.87 & -- & -- \\
\hline $14 / / 5 / 2014$ & 1.69 & -- & -- \\
\hline $10 / 6 / 2014$ & 2.51 & 2.62 & 2.52 \\
\hline $1 / 7 / 2014$ & 3.24 & 3.04 & 2.60 \\
\hline $24 / 7 / 2014$ & 2.79 & -- & -- \\
\hline
\end{tabular}

Fuente: elaboración propia

Tabla 4. Porcentaje de similitud de Sorensen (PS) en los puntos de muestreo

\begin{tabular}{|l|l|l|l|}
\hline Fecha & $\begin{array}{l}\text { PS Cortina - } \\
\text { Centro }\end{array}$ & $\begin{array}{l}\text { PS Cortina - } \\
\text { Cola }\end{array}$ & $\begin{array}{l}\text { PS Centro - } \\
\text { Cola }\end{array}$ \\
\hline $20 / 3 / 2014$ & $50 \%$ & $20 \%$ & $42 \%$ \\
\hline $3 / 4 / 2014$ & $91 \%$ & $83 \%$ & $78 \%$ \\
\hline $24 / 4 / 2014$ & - & 58 & - \\
\hline $10 / 6 / 2014$ & $88 \%$ & $23 \%$ & $24 \%$ \\
\hline $1 / 7 / 2014$ & $71 \%$ & $51 \%$ & $71 \%$ \\
\hline
\end{tabular}

Fuente: elaboración propia 


\section{DISCUSIÓN}

\section{Composición}

De acuerdo con Agüero (2013), la Organización Mundial de la Salud (OMS) establece que los niveles de alerta de algas tóxicas son los siguientes: nivel bajo $<20,000$ céls $/ \mathrm{mL}$, nivel moderado de 20,000 a 100,000 céls $/ \mathrm{mL}$ y nivel alto $>100,000$ céls $/ \mathrm{mL}$. Como se puede observar en la Tabla 1, se encuentra cerca del nivel moderado.

La comunidad de fitoplancton en el embalse estuvo dominada por Cyanophyceae $67 \%$, Coscinodiscophyceae $24 \%$ y Bacillariophyceae $2 \%$ para las diatomeas y las clorofitas con $5 \%$ de Chlorophyceae y $2 \%$ Zygnematophyceae, esta composición es parecida a la encontrada por Agüero (2013), para el Embalse Laureles, espacialmente se presentaron diferencias en la composición taxonómica por estaciones de muestreo, debido a la mayor presencia de Cianofíceas principalmente de Anabaena sp. 19,407 céls/mL, Cylindrospermopsis sp. 2,800 céls/mL, Nostoc sp. 382 céls $/ \mathrm{mL}$, para los valores pico y en menor cantidad Microcystis sp. 47 cels $/ \mathrm{mL}$ en la cortina. Según De León y Chalar (2003), la dominancia de formas filamentosas sobre las coloniales, indican condiciones turbulentas del sistema, situación que es muy común en este tipo de ecosistemas por la influencia de los drenajes provenientes de los tributarios.

Las diatomeas se registraron en el centro y la cola principalmente Aulacoseira sp. y Melosira sp.. Según De León y Chalar (2003), Aulacoseira sp. también es característica de sistemas turbulentos y sometidos a constantes cambios en la calidad de luz debido a sus movimientos a través del gradiente lumínico.

\section{Abundancia, biomasa y diversidad}

La abundancia del fitoplancton presentó dos etapas diferenciadas: 1 marzo - abril y 2 mayo - agosto. Durante la primera etapa la abundancia promedio fue de 920 céls $/ \mathrm{mL}$ característico de ambientes lenticos, mesotróficos a eutróficos (García y Anselmi, 1989; Calijuri y Tundisi, 1990; García, 1977; Izaguirre y Vinocur, 1994; Izaguirre et al., 1990, en De León y Chalar, 2003). Por el contrario en la segunda etapa la abundancia promedio fue de 220 céls $/ \mathrm{mL}$.

En cuanto a la biomasa fitoplanctónica medida como la concentración de clorofila a, se necesitan datos de un ciclo anual para obtener el grado de eutrofía del sistema, lo que se puede observar en la Figura 5 es la disminución en la concentración durante 
el invierno, y vuelve a incrementar en el mes de julio.

En cuanto a la diversidad, los registros más altos se presentaron inmediatamente después del verano en los meses de mayo hasta agosto, donde el centro del embalse es el sitio que presentó mayor diversidad por la predominancia de condiciones lóticas (Tabla 3). Con los resultados del porcentaje de similitud de Sorensen indican que, donde hay mayor similitud de las especies de fitoplancton es en la cortina - centro, esto se debe a que ambos puntos están más cercanos en el Embalse (Tabla 4).

Junto con la predominancia de las cianofíceas y perfiles de oxígeno disuelto clinógrado, según Ryding y Rast (1992), este perfil es causa de un aporte excesivo de nutrientes en las aguas profundas y según Wetzel (1981), por una descomposición excesiva de materia orgánica y sedimentos en el hipolimnion. Lo contrario es el perfil ortógrado donde el contenido de oxígeno es más alto en el hipolimnion característico de cuerpos de agua oligotróficos.

\section{CONCLUSIONES}

De acuerdo a los resultados obtenidos de los perfiles de oxígeno disuelto, la baja transparencia Secchi, la turbidez elevada en el hipolimnion, y el $67 \%$ de algas pertenecientes a la Clase Cyanophyceae, son características indicadoras de masas de agua eutróficas, esto permite concluir que las aguas del embalse Los Laureles, al momento de realizar el estudio, presentó características de un cuerpo de agua eutrófico.

Sin embargo, para obtener resultados con mayor grado de confiabilidad, se recomienda realizar los muestreos físicos, químicos biológicos y de productividad por lo menos cada quince días durante la época seca y lluviosa, aumentando los muestreos cuando se sospecha de floraciones algales, ya que estas relaciones son más precisas cuando los estudios se realizan por ciclos anuales, para identificar patrones de variación y tendencias que ayuden a prevenir y corregir este problema.

Lo ideal es realizar los monitoreos de fitoplancton de manera permanente, para lo cual se debiera contar con un profesional de la biología con experiencia en este tipo de trabajo, para poder realizar muestreos y análisis que logren detectar a tiempo las floraciones algales, y que presente alternativas de saneamiento o tratamiento del agua del embalse, y manejo de la cuenca a fin de disminuir la frecuencia y la abun- 
dancia de las algas de importancia sanitaria y reducir el riesgo en la salud de la población beneficiada con el preciado líquido.

\section{AGRADECIMIENTO}

Al Laboratorio de Control de Calidad de la División Metropolitana del SANAA, por proporcionar la infraestructura, el apoyo técnico y logístico. A la Universidad Nacional Autónoma de Honduras por haber brindado infraestructura y equipo en el Laboratorio de Hidrobiología. A la MSc. Isbela Hernández y Ph.D Mirna Marín por la revisión y enmiendas al documento.

\section{BIBLIOGRAFÍA}

Agüero, K. (2013). Estudio de la variabilidad en la población de fitoplancton presente en el Embalse Federico Boquín "Los Laureles". Informe final de práctica profesional supervisada. Servicio Autónomo Nacional de Acueductos y Alcantarillados (SANAA).Tegucigalpa, M.D.C. (47).

De León, L. y Chalar G. (2003). Abundancia y diversidad del fitolplancton en el Embalse de Salto Grande (Argentina - Uruguay). Ciclo estacional y distribución espacial. Limnetica 22(1-2): 103-113

Emanuelli, P. (2010). Metodología para la medición y evaluación de la biodiversidad en inventarios forestales. San Salvador. Cooperación Técnica Alemana (GTZ).

Luna, L., Díaz, B. y Rodríguez, J. (2010). Riesgo de contaminación con cianobacterias en tres embalses de Santiago de Cuba. Vol.14, n.2, pp. 0-0.

Martínez, G., Ortega, J. y Ramos, C. (2014). Biodiversidad del fitoplancton de aguas continentales en México. Revista Mexicana de Biodiversidad, Supl. 85:S54-S61. Moreno, J., Medina, C. y Albarracín, V. (2012). Aspectos Ecológicos y metodológicos del muestreo, identificación y cuantificación de cianobacterias y microalgas eucariotas. Reduca (Biología). Serie Microbiología. 5 (5): 110-125,

Novelo, E. (2011). Flora del Valle de Tehuacán-Cuicatlán, fascículo 90. Cyanoprokaryota J. komárek. México D. F.: Instituto de Biología UNAM. (96).

Novelo, E. (2012a). Flora del Valle de Tehuacán-Cuicatlán. Fascículo 102. Bacillariophyta Hustedt. Instituto de Biología UNAM: México D. F. Instituto de Biología UNAM. (230).

Novelo, E. (2012b). Flora del Valle de Tehuacán-Cuicatlán. Fascículo 94. Chlorophyta 
Pascher Instituto de Biología UNAM: México D. F. Instituto de Biología UNAM. (86).

Parra, O., M., Gónzales y V. Dellarossa. (1983ª). Manual taxonómico del fitoplancton de aguas continentales con especial referencia al fitoplancton de Chile. Chlorophyceae parte I. Concepción: Universidad de Concepción. (151).

Parra, O., M., Gónzales y V. Dellarossa. (1983b.) Manual taxonómico del fitoplancton de aguas continentales con especial referencia al fitoplancton de Chile. Chlorophyceae parte II. Concepción: Universidad de Concepción. (353).

Quirós, R. (2004) Cianobacterias en lagos y embalses de Argentina: década del 80. Buenos Aires: Universidad de Buenos Aires. (23).

Ryding, S. y Rast, W.(1992). El Control de la Eutrofización en Lagos y Pantanos. UNESCO. Ediciones Pirámide, S.A. Madrid, España. (375).

Rodríguez, I., Bustamante, A., Ruibal, L., Ruíz, A. y Busso F. (2008). Estudio del fitoplancton en el marco de monitoreo del Embalse San Roque (Cba). IV Congreso Argentino de Limnología, 27 al 31 de Noviembre de 2008. San Carlos de Bariloche: Instituto Nacional del Agua. (14).

Smith, R. y Smith, T. (2001). Ecología. 4ta Edición. Pearson Educación, S.A. Madrid, España. (642).

Tavera, M. (2013). Metodología para la gestión y planificación de un sistema de agua potable con suministro intermitente: aplicación a la ciudad de Tegucigalpa, Honduras. Tesis doctoral. Universidad Politécnica de Valencia. Valencia, España. (360).

UNESCO. (2009). Cianobacterias Planctónicas del Uruguay. Manual para la identificación y medidas de gestión. Sylvia Bonilla (editora). Documento Técnico PHI-LAC, N 16. Montevideo: UNESCO. (105).

Vila, I., Ilse, B. y V. Montecino. (1987). Abundancia y distribución temporal del fitoplancton en el Embalse Rapel, Chile Central. Revista Chilena de Historia Natural 60: 37-55.

Wetzel, R. (1981). Limnología. Barcelona: Ediciones Omega, S.A. (679).

Whitford, L. y S. Schumacher. (1984). A manual of fresh-water algae. N.C.: Raleigh Sparks Press. (337). 